This is an Accepted Manuscript of an article published by Taylor \& Francis in Attachment \& human development 2017 vol 19 no 5 pp. 463-486 on 30 May 2017, available online: http:// dx.doi.org/10.1080/14616734.2017.1330833.

Dhis document has been downloaded from TamPubutafi. The Institutional Repository of University of Tampere

Running Head: TRAUMA AND PRENATAL ATTACHMENT

\title{
War Trauma and Maternal-Fetal Attachment Predicting Maternal Mental Health, Infant Development, and Dyadic Interaction in Palestinian Families
}

\author{
1. Raija-Leena Punamäki \\ University of Tampere, School of Social Science and Humanities, Department of \\ Psychology,Tampere, Finland \\ and
}

University of Seville, Department of Evolution and Education Psychology, Seville, Spain

2. Sanna Isosävi

University of Tampere, School of Social Science and Humanities, Department of

Psychology,Tampere, Finland

3. Samir, R. Qouta

Islamic University Gaza, Department of Education and Psychology, Gaza City, Palestine

\section{Saija Kuittinen}

University of Tampere, School of Social Science and Humanities, Department of

Psychology,Tampere, Finland

5. Safwat Y. Diab

Al Quds Open University, Department of Educational Psychology, Gaza Strip, Palestine 


\section{Author Note}

Raija-Leena Punamäki, Sanna Isosävi, and Saija Kuittinen are at the School of Social Sciences and Humanities, Department of Psychology, University of Tampere, Tampere, Finland; Samir R. Qouta is at the Department of Psychology and Education, Islamic University Gaza, Gaza City, Palestine, and Safwat Y. Diab is at Department of Educational Psychology, Al-Quds Open University, Gaza Strip, Palestine

This study was supported by grants from the Academy of Finland (\#25021024) and Finnish Cultural Foundation. We are very grateful for the Palestinian mothers and infants who shared with us their important experiences. The study would not be possible without our excellent fieldworkers, we are deeply thankful for them.

Correspondence concerning this article should be addressed to Raija-Leena Punamäki, University of Tampere, Tampere, Finland. Email: raija-leena.punamaki@uta.fi 


\begin{abstract}
Optimal maternal-fetal attachment (MFA) is believed to be beneficial for infant wellbeing and dyadic interaction, but research is scarce in general and among risk populations. Our study involved dyads living in war conditions and examined how traumatic war trauma associates with MFA and which factors mediate that association. It also modeled the role of MFA in predicting newborn health, infant development, mother-infant interaction, and maternal postpartum mental health. Palestinian women from the Gaza Strip $(\mathrm{N}=511)$ participated during their 2nd trimester $(\mathrm{T} 1)$, and when their infants were 4 (T2) and 12 (T3) months. Mothers reported MFA (interaction with, attributions to, and fantasies about the fetus), social support, and prenatal mental health (posttraumatic stress disorder, depression, and anxiety) at $\mathrm{T} 1$, newborn health at $\mathrm{T} 2$, and the postpartum mental health, infant's sensorimotor and language development, and mother-infant interaction (emotional availability) at T3. Results revealed, first, that war trauma was not directly associated with MFA but that it was mediated through a low level of social support and high level of maternal prenatal mental health problems. Second, intensive MFA predicted optimal motherreported infant's sensorimotor and language development and mother-infant emotional availability but not newborn health or maternal postpartum mental health.
\end{abstract}

Keywords: mother-fetal attachment (MFA), war trauma, maternal mental health, infant development, emotional availability 
War Trauma and Maternal-Fetal Attachment Predicting Maternal Mental Health, Infant Development, and Dyadic Interaction in Palestinian Families

Real and perceived threat, danger, and stress activate the attachment system in order to maximize the sense of psychological and physical safety and feelings of security (Bowlby, 1969). Activated attachment and emotional bonding in pregnancy guarantee protection for the fetus and future baby through maternal preparation for her care-giving role and increased sensitivity to the fetus's needs and unique characteristics (Cranley, 1981; Habersaat et al., 2014). The protective function of maternal-fetus attachment (MFA) can be especially salient in the life-endangering conditions of war, military violence, and refuge that are, unfortunately, reality for millions of pregnant women (Akol, Caluwaerts, \& Weeks, 2016). It is thus timely to learn about the determinants and consequences of MFA among mother and infant dyads living in war areas.

Intensive MFA involves love, emotional affiliation, bonding, positive thoughts and attributions to the future baby, and health-considering behavior during pregnancy (Condon \& Corkindale, 1997; Cranley, 1981; Muller, 1992). We could not locate studies of the impact of maternal war trauma on MFA, but mothers with a history of childhood maltreatment have been found to face difficulties in creating MFA (Schwerdtfeger \& Goff, 2007). Research on MFA deserves criticism for testing the role of single determinants, such as risk pregnancies, mother's attachment style, or supporting networks separately and in relatively small samples (Maas, Vreeswijk, Braeken, Vingerhoets, \& van Bakel, 2014). It is agreed that intensive MFA contributes to good mother-infant interaction and infant wellbeing, and ultimately to the development of secure attachment (Brandon, Pitts, Denton, Stringer, \& Evans, 2009; Laxton-Kane \& Slade, 2002), but prospective studies are rare about the predictive value of MFA. Furthermore, analyzing both determinants and consequences of MFA simultaneously could provide a more comprehensive view of the role of MFA in the transition to parenthood (Brandon et al., 2009). The participants in the 
present study are Palestinian mothers who have experienced multiple traumatic events during major wars, military operations, and the military siege in the Gaza Strip. We examined how maternal war trauma might associate with MFA, and whether MFA predicts infant development and dyadic interaction during the first year of life.

\section{Determinants of MFA}

Mothers develop an attachment to their infants during pregnancy in order to provide security and to serve survival, stimulated by maternal responsibility for the fetus's wellbeing (Ivry, 2010; Laxton-Kane \& Slade, 2002). Prenatal attachment serves to prepare for motherhood, involving core developmental tasks of love and affection, differentiation between self and the fetus, interacting and communicating with the fetus, and attributing characteristics and intentions to him/her (Cannella, 2005; Cranley, 1981). There are salient shifts in pregnancy toward optimal motherhood, such as forgetting oneself in favor of the future baby and transforming from womanhood to motherhood (Gloger-Tippelt, 1983; Raphael-Leff, 1996), which are reflected in the intensity and quality of MFA. The term "intensity" refers here to the time dedicated to thinking and talking about the future baby, as well as feeling, touching, and dreaming about the fetus. The term "quality" refers to the tenderness, emotional closeness, and pleasure of interacting with the fetus or imagined future baby. Intensive and high quality MFA reflects successful adaptation to pregnancy (Alhusen, 2008; Muller, 1992).

Reasons for mothers avoiding intensive affiliation and emotional bonds with the fetus have been attributed to earlier miscarriages and risk pregnancies, resulting in the fear of losing the baby, expecting negative delivery experiences, and concerns for the safety of the newborn (Rafael-Leff, 1996). However, the research does not unanimously confirm that obstetric risks and earlier pregnancy-related stressors interfere with optimal development of MFA (Alhusen, 2008; Cannella, 2005; Laxton-Kane \& Slade, 2002). Studies have found comparative intensity and normative 
intensification of MFA across pregnancy among mothers with a history of miscarriage (Tsartsara, 2006) and among mothers and fathers with a history of perinatal loss (Armstrong, 2002).

Nonetheless, there is also evidence of earlier prenatal loss predicting lack or low intensity of MFA (Armstrong \& Hutti, 1998).

Pregnancy activates the mother's memories of her own early experiences of being cared for (Bowlby, 1988; Bretherton \& Munholland, 2008), and if the recollections are traumatic, she may face difficulties in forming an optimal MFA (Stern, 1995). Empirical findings are, however, discrepant concerning the effects of the mother's own childhood relations and attachment style in predicting MFA. A study of 156 pregnant women showed that mutually supportive and emotionally secure childhood relationships with both parents predicted intensive MFA (Wilson et al. 2000). Also, other studies have confirmed that the mother's secure attachment style predicted intensive MFA (Althusen, 2013; Mikulincer \& Florian, 1999). By contrast, a cross-sectional study of 41 pregnant women showed that emotional bonds with their own parents did not associate with MFA (Schwerdtfeger \& Goff, 2007). Interestingly, a Dutch community study (N = 351) of pregnant women found that maternal attachment security (self-reported) did not associate directly with MFA but was mediated through maternal personality characteristics such as emotional stability, extraversion, and agreeableness (Maas et al., 2014).

It is accepted that vital social networks and emotional support, especially from the spouse, contribute to maternal wellbeing (Orr, 2004) and to optimal MFA (Diniz, Volling, \& Koller, 2014; Maas et al., 2014; Van den Bergh \& Simons, 2009; Yarchesk et al., 2009). A study of Brazilian teenage mothers $(\mathrm{N}=49)$ confirmed that a high level of social support was associated with optimal MFA and that emotional support could even buffer the negative impact of depression on MFA (Diniz et al., 2014). Maas et al. (2014) confirmed that sufficient partner support, together with positive maternal personality and future child characteristics, predicted intensive MFA. 
Maternal prenatal mental health problems, especially depression, are expected to harm MFA (Alhusen, 2008; Cannella, 2005), but the research is not unanimous. Two representative studies among formerly infertile women found depression to predict generally low intensity, lack of normative intensification of MFA during pregnancy, and negative contents of MFA (Hart \& McMahon, 2006; Hjelmstedt, Widstrom, \& Collins, 2006). Further evidence shows that both trait anxiety (Hart \& McMahon, 2006) and pregnancy-related anxiety (Armstrong, 2002) impaired MFA. A study of North American low-income mothers $(\mathrm{N}=166)$ found that severe depressive symptoms significantly predicted low intensity of MFA (Alhusen, Gross, Hayat, Rose, \& Sharps, 2012). McFarland et al. (2011) confirmed that women with clinically diagnosed major depression showed a lower level of MFA than did non-depressed women $(\mathrm{N}=161)$. However, a prospective Swedish study $(\mathrm{N}=274)$ found minor and mixed associations between maternal depression and MFA. Interestingly, mothers with clinically significant depression reported especially high levels of physical contact with the fetus and awareness of fetal movement (Seimyr, Sjogren, WellesNystrom, \& Nissen, 2009). Along these lines, a meta-analysis of 14 studies found low size effects of the predictive power of anxiety and depression on MFA (Yarcheski et al., 2009).

\section{MFA, Infant Development, and Dyadic Interaction}

Relatively few longitudinal studies are available on MFA predicting early mother-infant interaction, parenting experiences, or infant development. We could find only Siddiqui and Hagglöf (2000), who assessed the infant-mother interaction objectively and found that MFA, especially fantasies about and affection for the fetus, predicted mothers' greater involvement with and stimulation of their 12-month-old babies. Findings from a prospective Australian study $(\mathrm{N}=150)$ showed that optimal MFA predicted mothers' sensitive attunement to infants' mental states and maternal capacity to view their babies as intentional agents with their own thoughts, conceptualized as mind-mindedness (McMahon, Camberis, Berry, \& Gibson, 2016). 
Concerning infant wellbeing and development, one study $(\mathrm{N}=91)$ showed that intensive MFA at the third trimester predicted positive infant mood at 8 months (Wilson et al., 2000). Another study found that intensive MFA at second trimester predicted infants' more advanced cognitive, linguistic, fine-motor, and sensorimotor development at 14-26 months, but only if maternal postpartum depression and attachment style were not controlled (Alhusen et al., 2012). In addition, Siddiqui and Hagglöf (2000) reported that MFA did not predict infant development, as indicated by attentive responses.

\section{Context of the Present Study}

The participating Palestinian mothers represent Middle Eastern Islamic culture. Activation of an attachment system of seeking and providing safety is considered universal, due to its biological roots and evolutionary functions (Bowlby, 1969; Shaver, Mikulincer, Alonso-Arbiol, \& Lavy, 2010). However, the emergence and expression of attachment behavior and its representations may vary considerable across individuals and groups (Raby, Cicchetti, Carlson, Egeland, \& Collins, 2013), cultural scripts, and environmental demands (Keller, 2013). In tandem with the latter view, research suggests that there is a cultural diversity of prenatal beliefs, expectations, parenting goals, rites, and communication with the unborn (Bravo \& Noya, 2014; Ivry, 2010). However, culturally informed or comparative studies are still lacking on the occurrence, content, or function of MFA (Seimyr et al., 2014). Two studies have reported confirmatory factor analyses (CFAs) on the dimensions of MFA in different national groups, finding both unidimensional (Gau \& Lee, 2003; Muller, 1992) and multidimensional (Siddiqui \& Hägglöf, 1999; Della Vedova, Dabrassi, \& Imbasciati, 2008) solutions. A five-dimensional structure of fantasy, interaction, affection, differentiation of self from fetus, and sharing of pleasure has been identified in Swedish and Polish (Seimyr et al., 2014; Siddiqui \& Hägglöf, 1999) and Italian samples (Barone, Lionetti, \& Dellgiulia 2014; Della Vedova et al., 2008). There are no studies available for ancient Middle Eastern cultures 
on the dimensionality of MFA, and therefore our study tested whether the five-dimensional structure of MFA is also valid for Palestinian women.

This study was conducted in the Gaza Strip, the Occupied Palestinian Territory (oPt), in 2013-2015. The participating women had earlier experienced a major war, the 2008/09 War on Gaza ("Operation Cast Lead," to use the Israeli military term), and when their infants were a few months olds, the 2014 War on Gaza ("Operation Protective Edge”) broke out. The 2008/09 war lasted 23 days, and the 2014 war lasted 54 days, the death tolls being 1,417 and 2,251, respectively. In the 2014 war, there were 11,231wounded and in 273,000 displaced Palestinians (UN Human Rights Council, 2015; UN OCHA, 2014).

Approximately two million Palestinians live in the Gaza Strip, which has been under Israeli military siege and international economic boycott since 2007. The reason for the collective punishment is the rule of the Hamas government, which is considered a security risk for Israel. The imposed blockade on the movement of people from the Gaza Strip and reduced delivery of goods, gas, and electricity have resulted in high poverty, lack of basic resources, and insecurity (World Bank, 2015). The split within the Palestinian Authority between the Fatah government in the West Bank and the Hamas government in Gaza further deteriorated the political situation and the future prospects of Palestinians. Studies show that the long-lasting poverty, lack of basic resources and political insecurity can have severe negative consequences on families' mental health, infant care and somatic health. For instance, as many as two thirds of parents and children have been found to suffer from posttraumatic stress disorder, PTSD and a third from depression after the major wars in Gaza (Palosaari, Punamaki, Diab, \& Qouta, 2013; Thabet, Abu Tawahina, El Sarraj, \& Vostanis, 2013) and infant mortality has increased during the years of military siege (Abed, 2016; Naim et al., 2013; Kalter, Khazen, Barghouthi, \& Odeh, 2008). 


\section{Research Questions}

Research is not available on the role of prenatal attachment in Middle Eastern Islamic cultures, and therefore we first examined whether the structure of the MFA concept among Palestinian mothers corresponds with the earlier findings in European countries. We expected to identify the five dimensions of fantasy, interaction, affection, differentiation of self from fetus, and sharing of pleasure (Siddiqui \& Hägglöf, 1999).

Some research suggests a mother's own traumatic childhood experiences to interfere with the optimal development of MFA (Schwerdtfeger, \& Goff, 2007), but knowledge is lacking about the role of other kinds of traumatic experience. Therefore, second, we examined how a mother's exposure to war trauma (human and material losses, witnessing horrors, and life threat) is associated with the intensity and quality of MFA. We hypothesized that severe war trauma has negative impacts on MFA, indicated by low intensity and negative contents. As other determinants of MFA, we modeled the role of pregnancy complications, available social support, and maternal mental health in pregnancy (symptoms of posttraumatic stress disorder [PTSD], depression, and anxiety). We hypothesized that the negative impact of war trauma on MFA is mediated through increased pregnancy complications, poor social support, and mental health problems.

Research is scarce on the multiple consequences of MFA. Therefore, third, in our prospective follow-up study among Palestinian mothers, we simultaneously tested whether the MFA predicts (a) newborn health (birth weight, gestational age, need for emergency care, Newborn Intensive Care Unit [NICU]), (b) maternal postpartum mental health, (c) infants' sensorimotor and language development, and (d) mother-infant interaction (emotional availability) at 12 months. The models were tested by using Structural Equation Modeling (SEM). 


\section{Method}

\section{Participants and Study Procedure}

The participants were 511 Palestinian women from the Gaza Strip who were in their 2nd trimester of pregnancy (T1), 481 women when their infants were 4 months (T2), and 454 when the infants were 12 months (T3). They were sequentially selected from all the pregnant women attending maternal clinics in 10 government primary health care centers (PHCCs) representing governorates in the North, Gaza City, Middle Area, Khan Youniss, and Rafah. The inclusion criteria were residence in the geographic area and being in the 2nd trimester of pregnancy.

The ethical board of The Palestinian Ministry of Health $(\mathrm{MoH})$ approved the study protocol and measurements. The participants were recruited in the 10 centers, and the T1 interviews were conducted on their premises, while the T2 and T3 interviews took place in the women's homes. They were informed about the study purposes, and they gave their written informed consent for participation. The average duration of the interview in the PHCCs was approximately 45 minutes and about 60 minutes in the participants' homes.

Ten fieldworkers with BA degrees in a relevant field and former experience in research work attended comprehensive training on the research procedures, interviewing skills, ethical rules, and home visits. The first and second authors provided the training and supervised the fieldwork in weekly consultative meetings. Data collection was carried out at three time points: AugustSeptember 2013 (T1), April-June 2014 (T2), and January-March 2015 (T3). The 2014 War on Gaza broke out in July, between the T2 and T3 data collections.

The dropout from $\mathrm{T} 1$ to $\mathrm{T} 2$ was $5.9 \%(\mathrm{~N}=30)$. Reasons for dropout were miscarriage $(\mathrm{N}=$ 10), errors in housing information $(\mathrm{N}=8)$, unwillingness $(\mathrm{N}=3)$ or inability $(\mathrm{N}=5)$ to participate, and infant death $(\mathrm{N}=4)$. The dropout was independent of the women's age, $(F(1,509)=0.019, p=$ ns. $)$, number of children $(F(1,509)=0.03, p=$ ns. $)$, job status $\left(\chi^{2}(3,511)=0.51, p=\right.$ ns. $)$, economic 
status $\left(\chi^{2}(3,511)=0.81, p=\mathrm{ns}.\right)$, war trauma $(\mathrm{F}(1,509)=0.36, p=\mathrm{ns}$.$) , and symptoms of$ depression $(F(1,509)=0.60, \mathrm{p}=\mathrm{ns}$.$) or \operatorname{PTSD}(F(1,509)=0.69, \mathrm{p}=\mathrm{ns}$.$) .$

The dropout rate from $\mathrm{T} 2$ to $\mathrm{T} 3$ was $11.2 \%$ from $\mathrm{T} 1(\mathrm{~N}=57)$, mostly due to failures to locate the whereabouts of displaced women $(10.8 \%, \mathrm{~N}=55)$ and death $(\mathrm{N}=2)$. The dropout at $\mathrm{T} 3$ was also independent of the women's age $(F(1,509)=0.95, p=$ ns. $)$, number of children $(F(1,509)=$ $0.47, p=\mathrm{ns})$, job status $(\chi(3,511) \square 0.89, p=\mathrm{ns}$.$) , economic status (\chi(3,511)=1.67 p=\mathrm{ns}$. $)$, war trauma at $\mathrm{T} 1(F(1,509)=0.08, p=\mathrm{ns}$.$) , and prenatal depressive (F(1,509)=0.09, p=\mathrm{ns}$.$) or PTSD$ $(F(1,509)=2.69, p=.10)$ symptoms. In addition, dropout was independent of newborn birth weight $(F(1,474)=0.15, p=$ ns. $)$, prematurity, $(F(1,463)=2.39, p=n s$.$) , and NICU and hospitalization$ $(F(1,463)=0.03, p=\mathrm{ns})$.

\section{Measurements}

Demographic factors. At T1, women reported their age, number of children, and length of marriage through open questions and described their education (basic education, including primary and secondary school; vocational training; college and university/polytechnic education) and employment situation (permanent work, part-time work, unemployed, works at home, student, or other). To indicate the family's financial situation, women estimated on 5-point scales the level of difficulty faced in paying bills and making the monthly income last. Their answers were combined into (1) No difficulties and sufficient means, (2) Some difficulties and insufficient means, and (3) Substantial economic difficulties.

Pregnancy complications at T1. Complications included diagnoses of six pregnancyrelated medical problems (high blood pressure, high blood sugar level, bleeding or early contractions, risk of miscarriage, abnormalities in ultrasound, and/or other problems: $1=$ yes; $0=$ no). A sum variable was constructed by counting yes answers. 
Newborn health at T2. Mothers reported the newborn birth weight and height, as well as need for NICU or later hospital admission. The NICU and later hospitalization variables were combined $(1=$ need for treatment; $0=$ no need for treatment $)$. Mothers also reported the infant's gestational age, and a dummy variable was created to indicate prematurity $(1=$ premature [gestational age $<37$ weeks]; $0=$ full-term infant born at gestational week 37 or later).

War trauma. The scale includes 28 typical war events during the 2008/2009 War on Gaza and subsequent military offensives. Seven events probed family targeted traumatic events (e.g., death and injury of family members, detention or separation in family), nine dealt with witnessing horrifying scenes (e.g., deaths and injuries in explosions, and shelling), six concerned material destruction (e.g., damaged property, loss of home, destroyed mosques), and six concerned life threat (e.g., being afraid of dying). The women reported whether they had experienced these events in the last five years (yes $=1$; no $=0$ ). For the present study, we constructed both a sum variable (counting all yes answers) and separate scores for family targeted trauma, witnessing horrors, material destruction, and life threat sum variables.

Prenatal attachment. The Prenatal Attachment Inventory (PAI) (Muller, 1993) involves 21 descriptions probing mothers' fantasies, emotions, interactions, and future plans in relation to the unborn child. Examples are "I wonder what the baby looks like now," "I get very excited when I think about the baby," "I feel love for the baby," "I enjoy feeling the baby move," "I imagine what part of the baby I'm touching," and "I plan the things I will do with my baby." Women answered on 4-point Likert scales how often they felt, thought, and behaved as described $(1=$ almost never; $4=$ almost always). Studies have mainly used the total sum variable and found it reliable and valid to predict infant development and mother-infant interactions (Pallant, Haines, Hildingsson, Cross, \& Rubertsson, 2014). In the present study, separate sum variables are based on the CFA of the underlying dimensions in our Middle Eastern sample. 
Social support. The measure is based on Schwarzer and Leppin's (1991) theoretical construct of source of support and help, and on Helgeson's (2003) measure of functional support. The tool measures the availability of social support from four sources (spouse, own family [parents and siblings], friends and relatives, other people) and four functions of social support (emotional, material and financial, information and guidance, and help with everyday tasks [i.e., meals, childcare, transportation]). Women were asked whom they could count out on to provide each kind of support and help when they needed it. They indicated the availability of support and help by marking yes (1) or no (0) concerning each alternative. For this study, four sum variables were constructed to indicate the source of social support by counting responses across the spouse, own family, friends and relatives and others; their scores thus varied between 0 and 4 .

PTSD symptoms at T1 and T3. PTSD symptoms were assessed at T1 and T3 by the Harvard Trauma Questionnaire (HTQ, Mollica \& Caspi-Yavin, 1991). Of the 30 items, 16 describe intrusive, avoidance, and hypervigilance symptoms, and 14 trauma-induced negative alterations in survivors' worldviews, emotions, and behavior. The scale was developed for multicultural use, and chosen for the present study due to its high reliability, validity, and appropriateness to ongoing traumatic encounters in the Palestinian context (Salo, Qouta, \& Punamäki, 2005; Qouta, Punamäki, \& El Sarraj, 2008). The women answered on a 4-point scale to what extent they had suffered the symptoms during the previous one month $(0=$ not at all; $3=$ severely). Total PTSD scales were constructed for T1 and T3, internal consistency being $\alpha=.89$ and $\alpha=.93$ respectively.

Depressive symptoms at T1 and T3. The Edinburgh Depression Scale (EDS) of 10 items (Cox, Holden, \& Sagovsky, 1987) describes depression-related feelings, thoughts, and behaviors using four levels of increasing severity. Women evaluated the extent to which the descriptions fit their moods during the previous two weeks. The EDS has been found to be reliable and valid in multiple samples (Bergink et al., 2011), including Arab women (Ghubash, Abou-Saleh, \& 
Daradkeh, 1997). Sum variables were constructed with Cronbach's $\alpha$ values of .78 (T1) and .89 (T3).

Pregnancy-related anxiety. At T1, the women reported their worries and anxieties related to pregnancy, using the 10-item Pregnancy Anxiety Scale (PAS) (Levin, 1999). The symptoms covered anxiety and fears about being pregnant, childbirth, and hospitalization. Women estimated how well their feelings and thoughts matched with the descriptions using a 5-point scale $(1=$ not at all; $5=$ a lot $)$. A total sum variable was constructed $(\alpha=.80)$.

Disassociation symptoms. At T3, the Psychological Dissociative States Scale (PDSS)

(Nijenhuis et al., 2003) was applied to indicate postpartum mental health on anxiety domain. The 15 descriptions involved depersonalization and derealization (e.g., feeling unreal, immersion in deep thought, or not recognizing oneself). Women estimated how commonly they had ever had these kinds of experience using a 6-point scale $(1=$ never; $6=$ all the time $)$. A sum variable was constructed $(\alpha=.77)$.

Infant sensorimotor and language development at T3. A 15-item questionnaire on infant's vocalization, language utterances, and fine and gross motor development was applied. It was based on the Minnesota Child Development Inventory (MCDI) (Kopparthi et al., 1991). The items reflect increasingly demanding skills (e.g., the 8-item scale for language: from "baby imitates repetitiously baby talk of mama, papa" to "baby combines two words"). The 4-item scale for fine-motor development gave also descriptions of skills, such as "baby can hold a toy/thing using both hands" and "baby can pick a small thing up using thumb and one finger." Examples of the 3-item motor development scale are "baby moves and walks by supporting him/herself" and "baby walks without support." Mothers estimated on a 3-point scale whether the infant had performed the task $(1=$ not observed; 2 = observed once or occasionally; 3 = observed many times, routinely). The MCDI has been validated and corresponds with Bayley Sensory Motor Skills in 8-16-month-olds in Western (Kopparthi et al., 1991) and Middle Eastern (Baherie, 2013) societies. The current 15-item scale has 
been validated for evaluation of early communication, language, and sensorimotor development in Europe (Lyytinen, Ahonen, Eklund, \& Lyytinen, 2000). For this study, a total sum score was constructed by adding the three scales, with high values thus indicating high skills.

Infant-mother interaction. A short version of the Emotional Availability-Self-Report (EASR Brief) (Biringen, Vliegen, Bijttebier, \& Cluckers, 2002) was used to evaluate infant-mother interaction at 12 months. The 28-item questionnaire describes, first, close and positive dyadic relationships (10 items, e.g., "My baby likes to be with me most of the time," "My baby seems to light up when she/he sees me," "I am usually in a good mood when with my baby"); second, distant and hard-to-soothe dyadic relationships (10 items, e.g., "My baby is 'cranky' most of the time," "My baby doesn't seem to notice when I come back into the room," "It is hard to soothe my baby and he (or she) seems to be distressed a lot"); and, third emotional scaffolding (8 items, e.g., "I try to see things from my baby's perspective"). Women estimated the extent to which the descriptions applied to her and her baby using a 5-point Likert scale ( $1=$ not at all; $5=$ completely). The EA-SRBrief self-assessment form is based on the observational Emotional Availability Scales and has been shown to correlate significantly with them (Vliegen, Luyten, \& Biringen, 2009). The EA-SRBrief has not been validated in Middle Eastern samples, and thus for the present study we ran a CFA to detect the three expected dimensions. However, the results revealed only two dimensions, namely, "Positive communication" and "Difficulty soothing the baby."

Translation. All instruments were used in Arabic. The war trauma and the PTSD questionnaires were ready available in Arabic (Isosävi et al., 2017). A bilingual researcher translated the scales of depressive, anxiety, and dissociation symptoms and mother-infant interaction from English to Arabic, and one member of the research team conducted a back translation to check for accuracy. The scale of infant language and sensorimotor development was translated and backtranslated from Finnish to Arabic by the research group. 


\section{Statistical Analyses}

Cross tables describe the distributions of demographic, war trauma, obstetric, and newborn variables. Concerning the first research question on the fit of the five-dimensional structure of the MFA to the present data, we used CFAs in AMOS SEM (AMOS 15.0 software; SPSS Framework Version). We chose the Maximum Likelihood (ML) estimation for the CFA because the values of the MFA questionnaire ranged between 1 and 5. CFA was also applied to check the dimensionality of the mother-infant interaction questionnaire, the EA-SR Brief.

SEM was applied to analyze the determinants of MFA (the second research question) and consequences of MFA (the third research question). The first model tested both the direct and mediated impacts of war trauma on MFA. As an exogenous variable, the latent war trauma construct (four manifest variables: family targeted trauma, witnessing horrifying scenes, material destruction, and life threat) was regressed directly to the latent construct of MFA (manifest variables are based on the results of the CFA). The model then tested the roles of endogenous latent constructs of social support (four manifest variables: support from spouse, own family, friends and relatives, and others), maternal mental health in pregnancy (three manifest variables: symptoms of PTSD, depression, and pregnancy-related anxiety), and the manifest variable of pregnancy complications in mediating the association between war trauma and the MFA latent construct.

The final model testing the consequences of MFA also included the first model (See Figure 1). The MFA latent construct was directly regressed on the latent constructs of newborn health (three manifest variables: low birth weight, early gestational age, need for treatment), mother-infant interaction (two manifest variables: positive communication and difficulty soothing the baby), maternal postpartum mental health (three manifest variables: symptoms of PTSD, depression, and dissociation), and infant sensorimotor and language development (three manifest variables: sensorimotor, fine-motor, and language skills). AMOS uses an ML method for obtaining estimates of the parameters and produces bias-corrected bootstrap confidence intervals to construct both direct and indirect 
effects (MacKinnon, Lockwood, Hoffman, West, \& Sheets, 2002). The residuals of the latent endogenous mental health constructs, as well as newborn health and infant development latent constructs, were allowed to covary to reflect shared sources of variance not included in the model.

The criteria for model fitness were non-significant $\chi^{2}$ value, $\chi^{2} / \mathrm{df}<2.00$, Comparative Fit Index (CFI) and Tucker-Lewis Index (TLI) above .90, and Root Mean Square Error of Approximation (RMSEA) below .06 (Bentler, 2007). We did not replace missing values in the final models because they were few and randomly distributed. However, modification indices (MI) information on covariates between estimates was based on data with regression imputation (full-information maximum likelihood [FIML] estimates on missing data).

\section{Results}

\section{Descriptive Statistics}

Table 1 presents demographic and obstetric information. Women were between 16 and 46 years of age (mean age $=24.77 \pm 5.55)$. All were in the 2 nd trimester of pregnancy (mean in weeks $=17.59 \pm 3.08)$, and $18 \%$ were expecting their first child. The education level was relatively high, as more than a quarter $(27 \%)$ had a university or polytechnic degree, which corresponds with Palestinian general statistics (World Bank, 2006). A large majority of the women worked at home, and about a half estimated their family's economic situation as poor, indicated by shortages of funds to cover everyday necessities and difficulty in paying bills.

Infant data showed that $18(5.4 \%)$ babies were born prematurely, $13 \%$ needed treatment in control or intensive care immediately after birth, and 9\% later. Very low birth weight (LBW) occurred in $8.4 \%$ of newborns, and about a half of them were in the $2500-3500 \mathrm{~g}$ weight range. It is noteworthy that not all prematurely born infants had a LBW, which explains the different percentages. 
War trauma involved experiences of family targeted trauma, witnessing horrors, material losses, and life threat, as shown in Table 2. Fear of death was the most common, with $90 \%$ of women believing that they were going to die, and $83 \%$ that they were actually close to death. About half $(56 \%)$ were evacuated from their homes due to warfare, had witnessed shelling of their neighborhood (56\%), or had witnessed people being killed (58\%). About a quarter (26\%) had witnessed friend/s being killed or/and had had a family member injured (26\%).

\section{Insert Table 2 about here}

\section{Dimensionality of MFA}

To answer the first research question about the structure of MFA, the results of the CFA on the 21 items of the PAI are presented in the Appendix. The SEM measurement model indicated that the MFA structure revealed in the Palestinian sample did not correspond with the earlier finding of five dimensions of fantasy, interaction, affection, differentiation of self from fetus, and sharing of pleasure (Siddiqui \& Hägglöf, 1999). Instead, the best solution of the CFA model involved three dimensions, referred to as (1) Interaction with fetus, (2) Attribution and sharing, and (3) Fantasy and preparation. The model had a good fit to the data $\left(\chi^{2} / \mathrm{df}=1.89 ; \mathrm{CFI}=.90\right.$, TLI $=.91$, RMSEA $=.042$ [90 \% CI: .034-.049]). Yet, $\chi^{2}(155)=293.21, p<.0001$ was significant, which is common in large samples (Yuan, Hayashi, \& Bentler, 2007). All standardized regression weights loaded significantly on one dimension only.

The interaction with the fetus dimension included items like "I share secrets with the fetus" or "I know the baby hears me." The attribution and sharing dimension consisted of items such as "I think the baby is already a personality" and "I tell others about things that my baby is doing inside me." The fantasy and preparation dimension involved items such as "I imagine calling the fetus by name" and "I buy/make things for the baby." 


\section{Measurement Models of Study Variables}

Table 3 shows the measurement models of the SEM analyses for determinants and consequences of MFA. The measurement model had a good fit to the data $\left(\chi^{2}(165)=301.43, p<\right.$ $.001 ; \chi / \mathrm{df}=1.86 ; \mathrm{CFI}=.90, \mathrm{TLI}=.91, \mathrm{RMSEA}=.056$ [90 \% CI: .050-.062]), although the $\left(\chi^{2}\right)$ statistic indicated misfit ( $<.001)$ due to large sample size (Yuan et al., 2007). Results confirmed significant standardized loading estimates of all manifest variables for the latent constructs, except for infant-mother interaction, indicated by two manifest variables (Positive communication and Difficulty soothing the baby: standardized $\beta$ loadings of .01 and $.02, p s=n s$.). Subsequently, we calculated a total sum score including both variables and re-scored the values so that a high level indicated positive infant-mother interaction. It was included as a manifest variable in the SEM analysis.

Insert Table 3 about here

\section{War Trauma and MFA}

To answer the second research question, we tested the direct and indirect impacts of traumatic war events on MFA. The SEM model showed good fit indices $\left(\chi^{2}(137)=217.43, p<.001 ; \chi^{2} / \mathrm{df}=\right.$ 1.80; CFI $=.95, \mathrm{TLI}=.93, \mathrm{RMSEA}=.040$ [90 \% CI: .028-.051]), although significant $\chi^{2}$ indicated misfit $(\mathrm{p}<.001)$. Figure 1 illustrated the results, although the values are from the final model. Against our hypothesis, the non-significant $\beta$ coefficient indicates that mother's severe exposure to war trauma was not negatively associated with MFA $(\beta=-.07, t=-0.77, p=.44)$. Instead, the mediating hypothesis was substantiated, as the association was mediated through social support and maternal prenatal mental health. Severe war trauma was associated with poor social support ( $\beta=-$ $.16, \mathrm{t}=-1.93, p<.05)$, which in turn was associated with less intensive MFA $(\beta=-.38, t=-3.32, p$ $<.0001)$. War trauma was also associated with a high level of maternal mental health problems $(\beta=$ $.56, t=7.56, p<.0001)$, which were then marginally associated with less intensive MFA $(\beta=-.16, \mathrm{t}$ $=-1.85, p<.06)$. Severe war trauma was associated with a higher level of pregnancy complications 
$(\beta=.19, \mathrm{t}=2.25, p<.05)$, but they did not further associate with MFA $(\beta=.-.01, t=-0.19, p=$ $.85)$.

The model of war trauma influencing MFA, including direct and indirect paths through social support, maternal mental health, and obstetric complications, explained $17 \%$ of the variance of MFA. The direct standardized effect of war trauma on MFA was lower $(\beta=-.07)$ than the mediating indirect effect $(\beta=-.15)$. Thus, available social support and maternal prenatal mental health significantly mediated the negative impact of traumatic war events on MFA, while obstetric complications were not significant.

Insert Figure 1 about here

\section{Consequences of MFA}

Figure 1 presents the final SEM model. It includes both determinants (war trauma, social support, maternal prenatal mental health, and pregnancy complications) and consequences of MFA (newborn health, maternal postpartum mental health, infants' sensorimotor and language development, and mother-infant interaction). The model had a good fit to the data $\left(\chi^{2}(225)=\right.$ 412.29, $p<.0001 ; \chi^{2} / \mathrm{df}=1.83 ; \mathrm{CFI}=.93, \mathrm{TLI}=.93, \mathrm{RMSEA}=.040$ [90 \% CI: .034-.047]), although the $\chi^{2}$ statistic was significant.

Findings confirmed that intensive MFA predicted significantly infants' good sensorimotor and language development $(\beta=.16, \mathrm{t}=2.32, p<.02)$ and positive infant-mother interaction indicated by emotional availability $(\beta=.12, \mathrm{t}=2.04, p<.04)$ at 12 months. MFA did not significantly predict maternal postpartum mental health problems $(\beta=-.08, \mathrm{t}=-1.38, p=.17)$ or newborn health $(\beta=.02, \mathrm{t}=0.32, p=.75)$.

\section{Discussion}

Developing attachment bonds may be highly challenging for mothers who attempt to protect their unborn babies and care for their infants in the midst of wars and life threats. Our results showed, however, that a mother's severe war trauma did not directly affect her attachment to the 
fetus (MFA) but that the negative impact was mediated through poor social support and maternal mental health problems. Earlier research has confirmed the beneficial role of good social support and maternal mental health for MFA (Hart \& McMahon, 2006; Yarchesk et al., 2009). Here, among war-affected mothers, sufficient prenatal support from family and good mental health contributed to intensive MFA, which in turn predicted both optimal mother-reported infant development and mother-infant interaction when the child was one year old. Our finding thus provides empirical evidence for the generally believed, but rarely studied favorable consequences of intensive MFA in the transition to parenthood.

A majority of studies have used MFA as a one-dimensional concept indicating intensity and quality of attachment to the future baby (Alhusen, 2008; Laxton-Kane, \& Slade, 2002). However, research on the structural elements has established the multidimensionality of MFA among women in South, North, and Eastern European countries (Barone et al., 2014; Bielawska-Batorowicz, \& Siddiqui 2008; Siddiqui \& Hägglöf, 1999). Our results revealed the three-dimensional structure of MFA among Palestinian women, which differs from the five-factor model found in European samples. Our findings identified dimensions of interaction with the fetus, attributing characteristics to him/her and sharing the experience with others, as well as fantasizing about the baby and preparation for parenting. Two main differences emerged between the current and earlier results. In our data, sharing the joy with other people loaded into the same dimension with attribution of fetal characteristics, whereas sharing was a separate dimension in the earlier studies. Among Palestinian women, the items of affection and love (e.g., "I love my baby" and "I enjoy feeling the baby move") loaded in all three dimensions. In the European samples, the affectional bond with the future baby seemed to be a separate aspect of MFA, whereas in our study it was embedded in the behavioral, cognitive, and physiological aspects of MFA. However, it is too early to interpret the difference to indicate that "a generalized affection and emotionality" characterizes ancient Middle Eastern cultures, whereas Western experiences of motherhood may be more framed and 
compartmentalized. Moreover, any comparison is difficult because our CFA was based on SEM, whereas earlier studies used a PCA that did not covary the error terms. It is noteworthy that only the first three factors in the five-factor solution of Siddiqui and Hägglöf (1999) produced reliable sum variables, while the three-factor model in our data showed good fit in the SEM measurement model. Mothers' experiences of severe war trauma did not interfere with their capacity to create an intensive attachment to the future baby. This result was contrary to our hypothesis and possibly also against intuitive expectations concerning mothers in life-threatening situations. It is accepted that pregnant women and infants are especially vulnerable to negative trauma impacts, indicated by obstetric, somatic, and mental health problems (WHO/UNICEFF, 2008). However, it may also be that traumatic experiences can strengthen a mother's motivation to protect new life. A Palestinian study revealed that among mothers, an accumulation of war trauma was associated with increased attachment and emotional availability to their school-age children, while among fathers the war trauma was associated with decreased attachment (Palosaari, Punamäki, Qouta, \& Diab, 2013). Although the current study did not reveal a strengthening but only the intactness of maternal prenatal attachment, the result confirms the significance of psychological processes in protecting children in war. Our hypothesis of negative trauma impacts on MFA was based on research on interpersonal trauma, such as childhood maltreatment, and thus future research should analyze whether the nature and timing of trauma are decisive for MFA.

We did not find earlier research analyzing psychosocial factors as possibly mediating the impacts of maternal trauma on MFA. Our results showed that the severe war trauma formed a risk for low intensity of MFA only if the trauma was associated with poor social support and with high levels of symptoms of PTSD, depression, and anxiety. It is accepted that help and support from family, spouse, and other significant people are important for pregnant women (Orr, 2004; Yarchesk et al., 2009), and our findings confirmed their critical role in the life-endangering conditions of war. Many aid programs for mothers and infants in war zones aim at enhancing 
nutrition, reproductive health, and general wellbeing (Casey, 2015; WHO/UNICEFF, 2008). Our results imply that preventive and treatment programs should integrate elements that improve prenatal maternal mental health and social support, and ultimately should enhance the infant-mother attachment relationship in both pre- and postnatal periods.

Our results confirmed the beneficial role of MFA in predicting maternal emotional availability and optimal infant sensorimotor and language development, as reported by the mother. The few available prospective studies have found either intensive MFA to predict good dyadic interaction but not infant development (Siddiqui \& Häglöf, 2000) or to affect infant characteristics but not dyadic interaction quality (Wilson et al., 2000). The present study differs from previous ones in using multilevel modeling of both determinants and consequences of MFA in a relatively large sample of women and infants. Parental attachment to a child serves multiple purposes: first, keeping him/her alive; second, knowing and reflecting the needs, emotions, and experiences of the child; and third, learning the mutual enjoyment of closeness and fear of separation (Bowlby, 1988). Prenatal attachment to the fetus apparently shares some similar purposes as those that Bowlby originally conceptualized for infant-parent attachment, although some researchers may disagree (Pollock \& Percy, 1999). This similarity may explain why loving, caring, and enjoyable bonding to the fetus contributed prospectively to the infant-mother interaction. Through prenatal attachment, mothers learn primarily to know the baby and can therefore more easily attune to the infant's temperamental and diurnal rhythms. In their prenatal attachments, mothers have dreamed about and prepared themselves to meet the child, which helps them discover adequate responses, synchronize intensity of dyadic emotional expressions, and enjoy reciprocal familiarity with the baby.

It is noteworthy that the MFA did not predict newborn health or maternal postpartum mental health, although it was important to infant-mother interaction and infant development. Our results thus suggest the outcome-specificity of MFA, but more research is needed on the underlying psychological and obstetric mechanisms that may transmit prenatal attachment to the infant's and 
parent's mutual early experiences and child development. Contemporary findings on fetal programming, that is, maternal stress and mental health affecting the fetus and later human development (O'Connor, Monk, \& Fitelson, 2014), make the question about multiple transmission dynamics even more relevant. In war conditions, material, economic, and political considerations are usually emphasized, while psychological wellbeing is considered a luxury (Summerfield, 2002). Our findings stress the need to also acknowledge psychological wellbeing, human attachment, and experiential aspects of family life, in addition to satisfying war survivors' basic and human rights needs.

In the current data, the indicators of newborn health (gestation maturity and birth weight) did not differ from epidemiological data among Arab Muslim women generally or from Palestinian statistics, except the need for intensive care and hospitalization being relatively high in our data (Roudi-Fahimi, 2003; Chavez et al., 2015). An earlier report based on the same data showed that maternal exposure to severe war trauma did not associate with newborn health (reference omitted for anonymity). It would be crucial to study psychosocial and obstetric paths and their interactions in predicting infant health and development in war conditions.

It is important to remember that there are two distinct approaches to conceptualizing the prenatal attachment phenomenon. The questionnaire approach, applied in the present study, involves emotional, cognitive, and behavioral dimensions of forming an attachment relationship or expressed emotional bond to the fetus, conceptualized as MFA. Attachment representations of the fetus, future baby, and oneself as a parent, assessed by interview methods, reveal parental inner working models, memories, and various states of mind that are more dynamic and comprehensive (Slade \& Cohen, 1995; Zeanah, Stafford, \& Zeanah, 2005). The MFA questionnaire produces information that is more conscious and thus also more vulnerable to social desirability, whereas coded interview material produces prenatal representations that also reveal semi- or unconscious views of the future baby and parenting. In contrast to the scarce prospective research on MFA, 
research on attachment representations has shown that balanced or functional (i.e., rich, coherent, flexible, and sensitive) prenatal representations predict secure infant-mother attachment (Dollberg, Feldman, \& Keren, 2010; Huth-Bocks, Levendosky, Bogat, \& von Eye, 2004; Zeanah et al., 2005). It would be intriguing to combine these two approaches to illuminate the multiple levels, distinct functions, and possible underlying mechanism of MFA and the representational approach to preparation for parenthood.

\section{Limitations of the Study}

The strengths of the study include relatively large sample size and small dropout number, while the main limitation is the exclusively single informant reporting. All measures are mother-reported, although objective testing, clinical interviews and observation data would have been preferable. It would have been more reliable to use registry and hospital data on prenatal obstetric and newborn health information. Similarly, video recordings and objective scoring of the quality of mother-infant interaction would have been preferable to mothers' own reports, which are open to social desirability. With regard to infant development, for instance, the Bayley IV scales would provide measurements that are more comprehensive and objective, but for practical reasons we had to rely on mothers' reports of their infants' sensorimotor and language development. In addition, clinical interviews would be more reliable than questionnaires with regard to the maternal mental health indicators comprising symptoms of PTSD, depression, anxiety, and dissociation. Finally, we are aware that an ideal objective documentation of a family's exposure to the war trauma would be more valid than mother-reported experiences. For instance, we could have photodocumented the extent of home destruction or checked the occurrence of human losses in the international archives, such as UN fact-finding missions (United Nations Human Rights Council, 2009). The self-reporting nature of the measures may have biased the results. Especially the 
findings of MFA predicting infant development and dyadic interaction must be interpreted with caution, because a more objective outcome assessment may change them.

To conclude: The contents, course, and intensity of MFA are highly informative with regard to the unique period of becoming a parent, even, or maybe especially, in the life-endangering conditions of war. Early interventions to enhance healthy transition to motherhood typically encourage intensive and balanced attachment representations and sensitive affiliation with the future baby (Slade et al., 2005). This is based on the premises that optimal MFA and attachment representations contribute to better maternal postpartum health, favorable infant development, and, in particular, to more enjoyable and more structured infant-mother interaction (Cicchetti, 2015; Laxton \& Slade, 2002; McFarland et al., 2011). Our findings supported these premises concerning mother-reported infant development and dyadic interaction, albeit with the caution caused by single informant reporting. 


\section{References}

Abed, Y. (2016). Risk factors associated with preterm birth in the Gaza Strip: Hospital-based casecontrol study. Eastern Mediterranean Health Journal, 13(5), 1132-1141. emro.who.int/emhj/1305/

Akol, A D, Caluwaerts, S, \& Weeks, A D. (2016). Pregnant women in war zones. British Medical Journal, 353. doi: 10.1136/bmj.i2037

Alhusen, J. L. (2008). A literature update on maternal-fetal attachment. Journal Obstetric, Gynecological and Neonatal Nursing, 37(3), 315-328. doi: 10.1111/j.15526909.2008.00241.x

Alhusen, J. L., Gross, D., Hayat, M. J., Rose, L., \& Sharps, P. (2012). The role of mental health on maternal-fetal attachment in low-income women. Journal of Obstetric, Gynecological and Neonatal Nursing, 41(6), E71-81. doi: 10.1111/j.1552-6909.2012.01385.x

Armstrong, D.. (2002). Emotional distress and prenatal attachment in pregnancy after perinatal loss. Journal of Nursing Scholarship, 34(4), 339-345

Armstrong, D., \& Hutti, M. (1998). Pregnancy after perinatal loss: The relationship between anxiety and prenatal attachment. Journal of Obstetric, Gynecologic, \& Neonatal Nursing, 27(2), 183189. doi: 10.1111/j.1552-6909.1998.tb02609.x

Baherie, A. (2013). Bayley Sensory Motor Skills. Cairo: Elinhada Egyptian Library. (In Arabic)

Barone, L., Lionetti, F. \& Dellagiulia, A. (2014). Maternal-fetal attachment and its correlates in a sample of Italian women: a study using the Prenatal Attachment Inventory. Journal of Reproductive and Infant Psychology, 32(3), 230-239. doi: http://dx.doi.org/10.1080/026 46838.2014 .883596

Bentler, P.M. (2007). On tests and indices for evaluating structural models. Personality and Individual Differences, 42(5), 825-829. doi: http://dx.doi.org/10.1016/j.paid.2006.09.024 
Bielawska-Batorowicz, E., \& Siddiqui, A. (2008). A study of prenatal attachment with Swedish and Polish expectant mothers. Journal of Reproductive and Infant Psychology, 26(4), 373-384. doi: $10.1080 / 02646830802426144$

Biringen, Z., Vliegen, N., Bijttebier, P., \& Cluckers, G. (2002). The Emotional Availability SelfReport. Requested from www.emotionalavailability.com.

Bowlby, J. (1969). Attachment, separation and loss. New York: Basic Books.

Bowlby, J. (1988). A secure base. Parent-child attachment and healthy human development. New York: Basic Books.

Brandon, A.R., Pitts, S., Denton, W.H., Stringer, C. A., \& Evans, H. (2009). A history of the theory of prenatal attachment. Journal of Prenatal \& Perinatal Psychology \& Health: APPPAH, 23(4), 201-209.

Bravo, I.M., \& Noya, M. (2014). Culture in prenatal development: Parental attitudes, availability of care, expectations, values, and nutrition. Child \& Youth Care Forum, 43(4), 521-538. doi: $10.1007 / \mathrm{s} 10566-014-9251-4$

Bretherton, I., \& Munholland, K. A. (2008). Internal working models in attachment relationships: Elaborating a central construct in attachment theory. In: Cassidy, J. \& Shaver, P. K. (Eds). Handbook of Attachment: Theory, Research, and Clinical Applications (2nd ed.), (pp. 103129). New York, NY: Guilford Press.

Cannella, B.L. (2005). Maternal-fetal attachment: an integrative review. Journal of Advanced Nursing, 50(1), 60-68. doi: 10.1111/j.1365-2648.2004.03349.x

Casey, S.E. (2015). Evaluations of reproductive health programs in humanitarian settings: a systematic review. Conflict and Health, 9(1), S1. doi: 10.1186/1752-1505-9-s1-s1

Cicchetti, D. (2015). Preventive intervention efficacy, development, and neural plasticity. Journal of American Academy of Child and Adolescence Psychiatry, 54(2), 83-85. doi: 10.1016/j.jaac.2014.11.006 
Chaves, S.C., Cecatti, J.G., Carroli, G., Lumbiganon, P., Hogue, , C.J., Mori, R..., \&Souza, J.P. (2015) Obstetric transtion in the World Health Organization multicountry surway on maternal and newborn health: exploring pathways for maternal mortality education. Revista Panamericana de Salud Publica, 37 (4/5), 2+3-210.

Cranley, M.S. (1981). Development of a tool for the measurement of maternal attachment during pregnancy. Nursing Research, 30(5), 281-284.

Della Vedova, A.M.D., Dabrassi, F., \& Imbasciati, A. (2008). Assessing prenatal attachment in a sample of Italian women. Journal of Reproductive and Infant Psychology, 26(2), 86-98. doi: $10.1080 / 02646830701805349$

Dollberg, D., Feldman, R., \& Keren, M. (2010). Maternal representations, infant psychiatric status, and mother-child relationship in clinic-referred and non-referred infants. European Child \& Adolescent Psychiatry, 19(1), 25-36. DOI 10.1007/s00787-009-0036-5

Field, T. (2011). Prenatal depression effects on early development: A review. Infant Behavior and Development, 34(1), 1-14. doi: http://dx.doi.org/10.1016/j.infbeh.2010.09.008

Gloger-Tippelt, G. (1983). A process model of the pregnancy course. Human Devevelopment, 26(3), 134-148.

Habersaat, S., Pierrehumbert, B., Forcada-Guex, M., Nessi, J., Ansermet, F., Müller-Nix, C., \& Borghini, A. (2014). Early stress exposure and later cortisol regulation: Impact of early intervention on mother-infant relationship in preterm infants. Psychological Trauma: Theory, Research, Practice, and Policy, 6(5), 457-464. doi: http://dx.doi.org/10.1037/a0033878

Hart, R., \& McMahon, C. A. (2006). Mood state and psychological adjustment to pregnancy. Archieves of Womens Mental Health, 9(6), 329-337. doi: 10.1007/s00737-006-0141-0 Helgeson, V.S. (2003). Social support and quality of life. Quality of Life Research,12: 25. Supplement. doi:10.1023/A:1023509117524 
Hjelmstedt, A., Widstrom, A. M., \& Collins, A. (2006). Psychological correlates of prenatal attachment in women who conceived after in vitro fertilization and women who conceived naturally. Birth, 33(4), 303-310. doi: 10.1111/j.1523-536X.2006.00123.x

Huth-Bocks, A. C., Levendosky, A. A., Bogat, G. A., \& von Eye, A. (2004). The impact of maternal characteristics and contextual variables on infant-mother attachment. Child Development, 75(2), 480-496. doi: 10.1111/j.1467-8624.2004.00688.x

Ivry, T. (2009). Embodying culture: pregnancy in Japan and Israel. New Brunswick: Rutgers University Press.

Kalter, H.D, Khazen, R.R., Barghouthi, M., \& Odeh, M. (2008). Prospective community $\square$ based cluster census and case $\square$ control study of stillbirths and neonatal deaths in the West Bank and Gaza Strip. Paediatric and Perinatal epidemiology, 22(4), 321-333.

Keller, H. (2013). Attachment and culture. Journal of Cross-Cultural Psychology, 44(2), 175-194. doi: $10.1177 / 0022022112472253$

Kopparthi, R., McDermott, C., Sheftel, D., Lenke, M.C., Getz, M., Frey, M. (1991). The Minnesota Child Development Inventory: validity and reliability for assessing development in infancy. Journal of Devevelopment and Behavior Pediatratry 2(4):217-222.

Laxton-Kane, M., \& Slade, P. (2002) The role of maternal prenatal attachment in a woman's experience of pregnancy and implications for the process of care, Journal of Reproductive and Infant Psychology, 20 (4), 253-266, DOI: 10.1080/0264683021000033174

Levin, J.S. (1991). The factor structure of the Pregnancy Anxiety Scale. Journal of Health and Social Behavior, 32, 368-381.

Lyytinen, P., Ahonen, T., Eklund, K., \& Lyytinen, H. (2000). Assessment of vocal and motoric development in infancy. Jyväskylä: Niilo Mäki Instituutti. (in Finnish). 
Koniak-Griffin, Deborah, Lominska, Susan, \& Brecht, Mary-Lynn. (1993). Social support during adolescent pregnancy: a comparison of three ethnic groups. Journal of Adolescence, 16(1), 43-56. doi: http://dx.doi.org/10.1006/jado.1993.1004

Maas, A.J.B.M., Vreeswijk, C.M.J.M., Braeken, J., Vingerhoets, A.J.J.M., \& van Bakel, H.J.A. (2014). Determinants of maternal fetal attachment in women from a community-based sample. Journal of Reproductive and Infant Psychology, 32(1), 5-24. doi:

$10.1080 / 02646838.2013 .853170$

MacKinnon, D. P., Lockwood, C. M., Hoffman, J. M., West, S. G., \& Sheets, V. (2002). A comparison of methods to test mediation and other intervening variable effects. Psychological Methods, 7(1), 83-104. doi: Doi 10.1037//1082-989x.7.1.83

McFarland, J., Salisbury, A. L., Battle, C. L., Hawes, K., Halloran, K., \& Lester, B. M. (2011). Major depressive disorder during pregnancy and emotional attachment to the fetus. Archi Womens Ment Health, 14(5), 425-434. doi: 10.1007/s00737-011-0237-z

McMahon, C., Camberis, A., Berry, S., \& Gibson, F. (2016). Maternal mind $\square$ mindedness: Relations with maternal-fetal attachment and stability in the first two years of life: Findings from an australian prospective study. Infant Mental Health Journal, 37(1), 17-28. DOI. 0.1002/imhj. 21548

Mikulincer, M., \& Florian, V. (1999). Maternal-fetal bonding, coping strategies, and mental health during pregnancy-The contribution of attachment style. Journal of Social and Clinical Psychology, 18(3), 255.

Muller, M. E. (1992). A critical review of prenatal attachment research. Scholarly Inquiry of Nursing Practice, 6, 5-22.

Muller, M.E, \& Mercer, R.T. (1993). Development of the prenatal attachment inventory. Western Journal of Nursing Research, 15(2), 199-215. 
Naim, A., Minutolo, R., Signoriello, S., \& Manduca P. (2013). Prevalence of birth defects in the Gaza Strip, occupied Palestinian territory, from 1997 to 2010: a pedigree analysis. The Lancet, 382(S27).

Nijenhuis, E. R. S., van Dyck, R., ter Kuile, M. M., Mourits, M. J. E., Spinhoven, P., \& van der Hart, O. (2003). Evidence for associations among somatoform dissociation, psychological dissociation, and reported trauma in patients with chronic pelvic pain. Journal of Psychosomatic Obstetrics and Gynecology, 24(2), 87-98.

O'Connor, T. G., Monk, C., \& Fitelson, E. M. (2014). Practitioner review: maternal mood in pregnancy and child development--implications for child psychology and psychiatry. $J$ Child Psychol Psychiatry, 55(2), 99-111. doi: 10.1111/jcpp.12153

Orr, S. T. (2004). Social support and pregnancy outcome: A review of the literature. Clinical Obstetetric Gynecology, 47(4), 842-855; Discussion 881-842.

Pallant, J. F., Haines, H. M., Hildingsson, I., Cross, M., \& Rubertsson, C. (2014). Psychometric evaluation and refinement of the Prenatal Attachment Inventory. Journal of Reproductive \& Infant Psychology, 32(2), 112-125. doi: 10.1080/02646838.2013.871627

Palosaari, E., Punamäki, R.L., Diab, M., \& Qouta, S. R. (2013). Intergenerational effects of war trauma among Palestinian families mediated via psychological maltreatment. Child Abuse and Neglect , 38, 114-122.doi:10.1016/j.chiabu.2013.04.006

Pollock, Philip H., \& Percy, Andrew. (1999). Maternal antenatal attachment style and potential fetal abuse. Child Abuse \& Neglect, 23(12), 1345-1357. doi: http://dx.doi.org/10.1016/S01452134(99)00101-5

Raby, K. L., Cicchetti, D., Carlson, E. A., Egeland, B., \& Collins, W. A. (2013). Genetic contributions to continuity and change in attachment security: a prospective, longitudinal investigation from infancy to young adulthood. Journal of Child Psychol and Psychiatry, 54(11), 1223-1230. doi: 10.1111/jcpp.12093 
Raphael-Leff, J. (1996). Pregnancy--procreative process, the "placental paradigm," and perinatal therapy. Journal of Americal Psychoanalytical Association, 44 Suppl, 373-399.

Roudi-Fahimi, Farzaneh. (2003). Women's reproductive health in the Middle East and North Africa: Population Reference Bureau Washington, DC.

Schwarzer, R. \& Leppin, A. 1991. Social support and health: A theoretical and empirical overview. Journal of Social and Personal Relationships, 8, 99-127.

Schwerdtfeger, K. L., \& Goff, B. S. (2007). Intergenerational transmission of trauma: exploring mother-infant prenatal attachment. Journal of Trauma Stress, 20(1), 39-51. doi: $10.1002 /$ jts.20179

Seimyr, L., Sjogren, B., Welles-Nystrom, B., \& Nissen, E. (2009). Antenatal maternal depressive mood and parental-fetal attachment at the end of pregnancy. Archieves of Women Mental Health, 12(5), 269-279. doi: 10.1007/s00737-009-0079-0

Shaver, P.R, Mikulincer, M., Alonso-Arbiol, I., \& Lavy, S. (2010). Assessment of adult attachment across cultures: Conceptual and methodological considerations. In P. Erdman, K.-M. Ng, \& S. Metzger (Eds.), Attachment: Expanding the Cultural Connections. (pp. 89-108). New York, NY: Routledge/Taylor \& Francis.

Slade, A., \& Cohen, L.J. (1996). The process of parenting and the remembrance of things past. Infant Mental Health Journal, 17, 217-238.

Slade, A., Sadler, L., De Dios-Kenn, C., Webb, D., Currier-Ezepchick, J., \& Mayes, L. (2005). Minding the baby: A reflective parenting program. The Psychoanalytic study of the child, 60(1), 74-100.

Stern, D. N. (1995). The motherhood constellation: A unified view of parent-infant psychotherapy. London: Karnac Books. 
Thabet, A.A., Abu Tawahina, A., El Sarraj, E., \& Vostanis, P. (2013). Death anxiety, PTSD, trauma, grief, and mental health of Palestinians victims of War on Gaza. Health Care: Current Reviews, 1:112. doi:10.4172/hccr.1000112

Tsartsara, E., \& Johnson, M. P. (2006). The impact of miscarriage on women's pregnancy-specific anxiety and feelings of prenatal maternal-fetal attachment during the course of a subsequent pregnancy: an exploratory follow-up study. Journal of Psychosomatics and Obstetric Gynaecology, 27(3), 173-182. PMID:17214452

UN-Human Rights Council (2015). The United Nations Independent Commission of Inquiry on the 2014 Gaza Conflict. A/HRC/29/52http://www.ohchr.org/EN/HRBodies /HRC/CoIGaza Conflict /Pages /ReportCoIGaza.aspx

UN-OCHA (2014). Occupied Palestinian Territory. Gaza Emergency Situation Report. 4 September 2014. United Nations Office for the Coordination of Humanitarian Affairs, OCHA, https://www.ochaopt.org/documents/ocha opt sitrep 0409 2014.pdf.

United Nations Human Rights Council, UNHRC (2009). Human rights in Palestine and other occupied Arab territories: Report of the United Nations fact-finding mission on the Gaza conflict. New York: UN General Assembly. Retrieved from http://www2.ohchrorg/english/bodies/hrcouncil/docs/12session/A-HRC-12-48.pdf.

Van den Bergh, B., \& Simons, A. (2009). A review of scales to measure the mother-foetus relationship. Journal of Reproductive and Infant Psychology, 27(2), 114-126. doi: $10.1080 / 02646830802007480$

Wilson, M. E., White, M. A., Cobb, B., Curry, R., Greene, D., \& Popovich, D. (2000). Family dynamics, parental-fetal attachment and infant temperament. Journal of Advanced Nursing, 31(1), 204-210. 10.1046/j.1365-2648.2000.01245.x 
WHO/UNICEF (2008). Strengthening action to improve feeding of infants and young children 6-23 months of age in nutrition and child health programmes. http://www.who.int/maternal_ child_adolescent /documents /978924159789.

World Bank (2006).West Bank and Gaza education sector analysis: Impressive achievements under harsh conditions and the way forward to consolidate a quality education system. Washington D.C.: The World Bank.

World Bank (2015). Economic monitoring report to the ad hoc liaison committee. Retrieved from http://documents.worldbank.org/curated/en/5631 81468182960 504/pdf/96601-REVISEDWP-Box391464B-AHLC-May-21-Book-fix-footnotes.pdf

Vliegen, N., Luyten, P., \& Biringen, Z. (2009). A multimethod perspective on emotional availability in the postpartum period. Parenting: Science and Practice, 9, 228-243. doi:10.1080/15295190902844514

Voegtline, K.,\& Stifter, C. (2010). Late-preterm birth, maternal symptomatology, and infant negativity. Infant Behavior \& Development, 33., (4), 545-554.http:// dx.doi.org /10.1016/j.infbeh.2010.07.006

Yuan, K.H., Hayashi, K., \& Bentler, P.M. (2007). Normal theory likelihood ratio statistic for mean and covariance structure analysis under alternative hypotheses. Journal of Multivariate Analysis, 98(6), 1262-1282. doi: http://dx.doi.org/10.1016/j.jmva.2006.08.005

Yarcheski, Adela, Mahon, Noreen E., Yarcheski, Thomas J., Hanks, Michele M., \& Cannella, Barbara L. (2009). A meta-analytic study of predictors of maternal-fetal attachment. International Journal of Nursing Studies, 46(5), 708-715. doi: http://dx.doi.org/10.1016/j.ijnurstu.2008.10.013

Zeanah, P. D., Stafford, B., \& Zeanah, C. H. (2005). Clinical interventions to enhance infant mental health: A selective review. UCLA Center for Healthier Children, Families and Communities. 
Table 1

Background, Obstetric, and Newborn Characteristics of the Sample

Participants $^{\mathrm{a}}$

$\% \quad \mathbf{n}$

\begin{tabular}{ccc}
\hline Age (years) & & \\
$16-20$ & 26.6 & 136 \\
$21-30$ & 53.6 & 274 \\
$31-40$ & 18.2 & 93 \\
$41-46$ & 1.6 & 8
\end{tabular}

Number of children

Expecting first child

$18.1 \quad 90$

$1-3$

$58.9 \quad 292$

$4-6$

$20.0 \quad 99$

$7-11$

3.0

15

Education

No formal education

1.26

Elementary school

$11.4 \quad 58$

Secondary school

55.6

284

High school

$3.3 \quad 17$

Vocational school

2.2

10

Polytechnic college/University

26.6

136

Length of marriage (years)

$<1$ year

$8.8 \quad 45$

$1-5$

45.2

231

6-10

26.4

135

11-20

17.6

90

$>20$ years

1.8

9

Economic situation: Income sufficiency ${ }^{\mathrm{a}}$

Enough money

45.4

232

Not enough to cover expenses

54.6

279

Economic situation: Difficulty with bills ${ }^{\mathrm{a}}$

None or little

59.1

302

Some or extensive

40.9

209

$\%$

n 


\begin{tabular}{lcc}
\hline Pregnancy complications (Yes answers) & & \\
High blood pressure & 2.5 & 13 \\
Abnormalities in ultrasound & 1.4 & 7 \\
Early delivery symptoms & 2.2 & 11 \\
Risk of miscarriage & 6.7 & 34 \\
$\quad$ Diagnosis of abnormalities & 5.3 & 27 \\
Other risks & 8.4 & 43 \\
Child's sex & & \\
Girl & 45.7 & 219 \\
Boy & 54.3 & 260 \\
Gestational age (weeks) & & \\
<37 & 5.4 & 18 \\
37 & 94.6 & 318 \\
Birth weight (g) & & \\
<2500 & 8.4 & 43 \\
2500-3499 & 54.6 & 279 \\
3500-4499 & 28.4 & 145 \\
> 4500 & 1.8 & 9 \\
Birth height (cm) & & \\
25-44.5 & 4.1 & 18 \\
45-54.5 & 85.5 & 376 \\
55-65 & 10.5 & 46 \\
Newborn health & & \\
Normal: returned home with the mother & 85.8 & 406 \\
Problematic: stayed in control unit & 11.2 & \\
Severe: stayed in NICU & 3.0 & \\
Baby's health later & & \\
Good & 90.7 & \\
Problematic; admitted to hospital & 9.3 & \\
\hline & & \\
\hline
\end{tabular}

${ }^{\text {a }}$ Participant numbers differed due to missing data 
TRAUMA AND PRENATAL ATTACHMENT

Table 2 Exposure to Traumatic War Experiences (\% and Frequencies)

\begin{tabular}{|c|c|c|}
\hline & \multicolumn{2}{|c|}{ Participants ${ }^{\text {a }}$} \\
\hline & $\%$ & $\mathrm{n}$ \\
\hline \multicolumn{3}{|l|}{ Family targeted trauma } \\
\hline Death of family member & 18.3 & 93 \\
\hline Injury of family member & 26.0 & 132 \\
\hline Detention of family member & 9.6 & 49 \\
\hline Shelling on own home & 19.6 & 99 \\
\hline Evacuation from home & 55.9 & 284 \\
\hline Death of relative/s & 38.9 & 197 \\
\hline Exposure to phosporous bombs & 23.2 & 118 \\
\hline \multicolumn{3}{|l|}{ Wittnessing horrors } \\
\hline Seen fried/s killed & 26.4 & 134 \\
\hline Seen friend/s injured by bombs/shells & 27.8 & 141 \\
\hline Seen other people killed & 57.7 & 292 \\
\hline Seen other people injured & 56.8 & 288 \\
\hline Watching a close person in pains & 33.7 & 171 \\
\hline Watching strangers in pains in ruins & 28.4 & 144 \\
\hline Witnessing shelling of neighborhood & 56.4 & 286 \\
\hline Witnessing fire and burning & 77.0 & 391 \\
\hline Witnessing explosions & 56.9 & 288 \\
\hline \multicolumn{3}{|l|}{ Material destruction } \\
\hline Lost belongings due shelling/bombing & 31.1 & 158 \\
\hline Damage to property & 28.0 & 142 \\
\hline Financial and income loss & 59.8 & 304 \\
\hline Destruction of home & 19.7 & 100 \\
\hline Car bomb-caused destruction & 69.9 & 355 \\
\hline Other material losses & 37.0 & 188 \\
\hline \multicolumn{3}{|l|}{ Life threat } \\
\hline Was close to loss of life & 83.3 & 423 \\
\hline Was in shock after shelling/bombing & 88.2 & 447 \\
\hline Believe that is going to die & 89.8 & 454 \\
\hline Near miss as a targeter and/or shelled & 69.0 & 350 \\
\hline Continuous fear of death & 57.4 & 291 \\
\hline Threatened by gun firing & 15.6 & 79 \\
\hline
\end{tabular}

Notes: ${ }^{\text {a }}$ The response rate ranged between 505-508; the percentages and frequencies refer to of yes-answers in the dichotomy scale. 
TRAUMA AND PRENATAL ATTACHMENT

Table 3

Loading Estimates of Manifest Variables for Latent Constructs of the SEM Models in Pregnancy, and Postpartum Four and Twelve Months

\begin{tabular}{|c|c|c|c|c|}
\hline \multirow[b]{2}{*}{ Observed variables for latent constructs } & \multicolumn{4}{|c|}{ Regression weights $^{\text {a }}$} \\
\hline & Unstd $\square$ & StdE & Std $\beta$ & t-tests ${ }^{b}$ \\
\hline \multicolumn{5}{|l|}{ War trauma } \\
\hline Family targeted & .70 & .09 & .54 & $8.05 * * *$ \\
\hline Witnessing horrors & 1.00 & & .64 & \\
\hline Material destruction & 0.83 & 0.10 & .69 & $8.29 * * *$ \\
\hline Life threat & 0.45 & 0.07 & .37 & $6.20 * * *$ \\
\hline \multicolumn{5}{|l|}{ Maternal-fetal attachment } \\
\hline Interaction with fetus & .94 & .09 & .60 & $10.41 * * *$ \\
\hline Attributions and sharing & 1.00 & & .68 & \\
\hline Fantasy and preparation & 1.28 & .13 & .79 & $10.22 * * *$ \\
\hline \multicolumn{5}{|l|}{ Social support } \\
\hline Spouse & 1.00 & & .52 & \\
\hline Family (parents and siblings) & 1.01 & .16 & .52 & $6.44 * * *$ \\
\hline Friends and relatives & .76 & .12 & .59 & $6.56 * * *$ \\
\hline Others & .53 & .09 & .46 & $6.11 * * *$ \\
\hline \multicolumn{5}{|l|}{ Maternal mental health in pregnancy } \\
\hline PTSD symptoms & 1.00 & & .86 & \\
\hline Depressive symptoms & 0.30 & 0.06 & .55 & $4.64 * * *$ \\
\hline Anxiety symptoms & 0.28 & 0.06 & .34 & $4.34 * * *$ \\
\hline \multicolumn{5}{|l|}{ New-born health } \\
\hline Birth weight & 1.00 & & .34 & \\
\hline Premature delivery & 1.14 & 0.53 & .50 & $2.13 *$ \\
\hline Need for intensive care or hospitalization & 1.84 & 0.79 & .35 & $2.02 *$ \\
\hline \multicolumn{5}{|l|}{ Maternal mental health postpartum } \\
\hline PTSD symptoms & 1.00 & & .83 & \\
\hline Depressive symptoms & 0.26 & 0.03 & .48 & $8.95 * * *$ \\
\hline Dissociative symptoms & 0.83 & 0.08 & .82 & $10.59 * * *$ \\
\hline \multicolumn{5}{|l|}{ Infant developmental at 12 months } \\
\hline Sensorimotor development & 0.60 & 0.04 & .75 & $16.66^{* * *}$ \\
\hline Fine motor skills & 0.86 & 0.05 & .82 & $17.41 * * *$ \\
\hline Language skills & 1.00 & & .83 & \\
\hline \multicolumn{5}{|l|}{ Infanf-mother interaction ${ }^{\mathbf{c}}$} \\
\hline Positive communication & 1.00 & .20 & .02 & \\
\hline Difficulty to soothe & .04 & .21 & .01 & 0.78 \\
\hline
\end{tabular}

Notes: $* \mathrm{p}>.05 ; * * * \mathrm{p}<.001$

${ }^{\mathrm{a}} \mathrm{N}=511$ at $\mathrm{T} 1 ; \mathrm{N}=481$ at $\mathrm{T} 2$ and $\mathrm{N}=454$ at T3. ${ }^{\mathrm{b}}$ Absence of StdE (Standard error) and t-values refer to parameters fixed to 1 in the measurement models; ${ }^{\mathrm{c}}$ The Infant-mother interaction (Emotional availability) did not reach significant weights 
TRAUMA AND PRENATAL ATTACHMENT

Appendix: CFAs for MFA based on SEM measurements (Prenatal Attachment

Questionnaire, 21 items)

Standardized Regression Weights

Questionnaire items

I II III t-values $^{\mathrm{b}}$

I Interaction with fetus

9. I imagine what part of the baby I'm touching .565

1.000

10. I know when the baby is asleep

.464

$6.96 * * *$

11. I can make my baby move

.508

$7.34 * * *$

17. I know why the baby is moving

.477

$7.07 * * *$

19. I share secrets with the baby

.458

$6.90 * * *$

20. I know the baby hears me

.470

$7.01 * * *$

\section{Attribution and sharing}

1. I wonder what the fetus looks like now

3. I enjoy feeling the baby move

4. I think that my baby already has a personality

5. I let other people put their hands on my tummy to feel the baby move

8. I tell others about the things the baby is doing inside me

15. I like to sit with my arms around my tummy

21 . I get very excited when I think about the baby

III Fantasy and preparation

2. I imagine that I call the fetus by name

$.451 \quad 1.00$

6. I know that things I do make a difference to the baby

7. I plan for the things I am going to do with my baby

12. I buy/make things for the baby

$.3875 .82 * * *$

13. I feel love for the baby

$.1753 .10^{* *}$

14. I try to imagine what the baby is doing in there

$.4936 .69 * * *$

16. I dream about the baby

$.4166 .08 * * *$

Notes: $* * \mathrm{p}>.01 ; * * * \mathrm{p}<.001{ }^{\mathrm{a}} \mathrm{N}=511$, missing values replaced by regression imputation based on ML estimation in AMOS ${ }^{\mathrm{b}}$ Absence of Standard Error (StdE) and t-values refer to parameters fixed to 1 in the measurement models. 




Pregnancy (T1)

Infant 4 months (T2) Infant 12 months (T3)

Figure 1. Structural equation model (SEM) results of determinants and consequences of maternalfetal attachment (MFA).

Nonsignificant paths, error terms, and correlated errors are not shown for clarity reasons (significant correlations were between PTSD and depression manifest variables at T1 and T3, and between prematurity and sensorimotor manifest variables). Path coefficients represent standardized $\beta$-values. For description of manifest variables and fixed estimates, see Table 3.

${ }^{*} p>.05 ;{ }^{* * *} p<.001$. 